
vol. 55, no. 6, pp. 1-4, June 2019, Art no. 7400804, doi: 10.1109/TMAG.2019.2897253.

\title{
A Perfectly Matched Layer for the Nonlinear Dispersive Finite-Element Time-Domain Method
}

\author{
David S. Abraham ${ }^{1}$, Student Member, IEEE, and Dennis D. Giannacopoulos ${ }^{1}$, Senior Member, IEEE \\ ${ }^{1}$ Department of Electrical \& Computer Engineering, McGill University, Montréal, Québec H3A 0E9, Canada
}

\begin{abstract}
A novel implementation of a Perfectly Matched Layer (PML) is presented for the truncation of Finite-Element Time-Domain (FETD) meshes containing electrically complex materials, exhibiting any combination of linear dispersion, instantaneous nonlinearity, and dispersive nonlinearity. Based on the complex coordinate stretching formulation of the PML, the presented technique yields an artificial absorbing layer whose matching condition is independent of material parameters. Moreover, by virtue of only modifying spatial derivatives, the incorporation of the PML into existing solvers for complex media is simple and straightforward. The resulting Material Independent PML (MIPML) is incorporated into a nonlinear dispersive method for the vector wave equation, which leverages the z-transform and Newton-Raphson techniques to yield an implementation free from recursive convolutions, auxiliary differential equations, and linearizations. This permits the unprecedented truncation and attenuation of nonlinear phenomena, such as spatial and temporal solitons, within the FETD method.
\end{abstract}

Index Terms-Dispersive Media, Finite-Element Methods, Nonlinear Media, Perfectly Matched Layers.

\section{INTRODUCTION}

D UE to limited computational resources, any problem treated numerically must be solved over a finite domain. However, when studying electromagnetic wave phenomena, the required domain may be infinite in extent, or the fields of interest may occur only within some small subset of a larger domain. In either case, the ideal result is a numerical truncation which mimics an infinite or much larger medium, suppressing any reflections at the boundary, and leaving the solution on the interior unchanged.

While many techniques have been devised to truncate computational domains, arguably one of the most successful has been that of Perfectly Matched Layers (PML). First derived by Berenger [1], the PML is a region of artificial attenuation which, as the name implies, is perfectly matched to the incident medium, thereby suppressing reflections and mimicking an infinite, or much larger, domain.

Berenger's initial PML introduced artificial electric and magnetic conductivities in such a way as to be reflectionless to the incident medium, while attenuating propagating waves. More specifically, in order to have the PML be reflectionless, it was necessary for the electric and magnetic conductivities to be related via a matching condition:

$$
\frac{\sigma_{E}}{\epsilon}=\frac{\sigma_{H}}{\mu}
$$

where $\sigma_{E}$ and $\sigma_{H}$ are the artificial electric and magnetic conductivities, and $\epsilon$ and $\mu$ are the permittivity and permeability. While simple in the case of linear media, the above matching condition substantially complicates the implementation when

Manuscript received October 28th, 2018. Revised, accepted, published. Corresponding author: D. S. Abraham (email: david.abraham@mail.mcgill.ca).

Color version of one or more of the figures in this paper are available online at http://ieeexplore.ieee.org.

Digital Object Identifier (inserted by IEEE). applied to electrically complex materials, in which the permittivity is a function of time, field strength, or both.

Further investigation later revealed additional matching conditions that can yield perfect transmission, and which do not depend on $\epsilon$ and $\mu$. Indeed, this became particularly evident when Berenger's original PML was reformulated in terms of an anisotropic (uniaxial) medium [2], or as a complex coordinate stretching [3], [4]. The resulting family of methods are sometimes known as Material Independent PMLs (MIPML) and are ideal for truncating nonlinear, dispersive, and even anisotropic media.

Some of these MIPML formulations have already seen application to nonlinear and dispersive Finite-Difference TimeDomain (FDTD) simulations [5], [6]. However, they have not been used for the Finite-Element Time-Domain (FETD) method yet.

In this paper, a coordinate stretching-based MIPML formulation for the FETD method will be derived and implemented for the truncation of computational domains containing general nonlinear dispersive media. The coordinate stretching approach is shown to yield a simpler more effective formulation than a split-field or uniaxial PML implementation. Furthermore, by leveraging the nonlinear Newton-Raphson FETD method, as well as the z-transform technique, the resulting FETD MIPML scheme is accurate, stable, scalable, and free of recursive convolutions, auxiliary differential equations, or linearizations. Lastly, the method's effectiveness is demonstrated via the absorption of a temporal soliton propagating in a dielectric slab waveguide.

\section{Electrically Complex Media}

Electrically complex materials play a vital role in many areas of science and technology, particularly in the field of nonlinear optics. Here, the complex nonlinear and dispersive properties of materials are often leveraged to facilitate or 
improve telecommunications infrastructure. As a result, the ability to accurately simulate and characterize these materials is paramount.

In the case of a dispersive medium, the permittivity is frequency dependent, which translates to a convolution between the permittivity and electric field in the time-domain. In the nonlinear case, the permittivity is a function of the electric field strength. These interactions are generally characterized via the material's polarization density vector, $\vec{P}$, for which an adequate model is often given by the following [7]:

$$
\vec{P}=\epsilon_{0} \chi^{(1)}(t) * \vec{E}+\epsilon_{0} \chi^{(3)}\left(\alpha E^{2}+(1-\alpha) g(t) * E^{2}\right) \vec{E}
$$

in which the susceptibility $\chi^{(1)}$ models linear dispersion, $\chi^{(3)}$ an instantaneous Kerr and/or stimulated Raman nonlinearity (with the $\alpha$ term controlling their relative strengths), and $*$ denoting convolution.

\section{DERIVATION}

The coordinate stretching formulation of the PML begins by expressing Faraday's and Ampère's laws in time-harmonic form within the PML medium as follows [3]:

$$
\begin{aligned}
\nabla_{B} \times \vec{E} & =-j \omega \vec{B} \\
\nabla_{D} \times \frac{1}{\mu} \vec{B} & =j \omega \vec{D}
\end{aligned}
$$

in which the curl operators have had their spatial derivatives modified, or stretched, such that:

$$
\frac{\partial}{\partial \xi} \mapsto \frac{1}{s_{\xi}} \frac{\partial}{\partial \xi}
$$

with:

$$
s_{\xi}=1+\frac{\sigma_{\xi}}{j \omega} .
$$

Note that the subscripts $B$ and D in (3) and (4) allow for different stretching factors to be applied to each field. Combining (3) and (4) and converting to the time-domain results in the following vector wave equation:

$$
\nabla_{D} \times \frac{1}{\mu} \nabla_{B} \times \vec{E}+\frac{\partial^{2}}{\partial t^{2}}\left(\epsilon_{0} \vec{E}+\vec{P}\right)=-\frac{\partial \vec{J}}{\partial t}
$$

where it must be noted that the curl operators are now timedependent and implicitly require convolution with $\vec{E}$.

Following the procedure outlined for nonlinear dispersive FETD in [8] and [9], a variational method can then be applied to yield a semi-discrete system:

$$
\frac{\partial^{2}}{\partial t^{2}}\left(\epsilon_{L} *[\tilde{T}]\{e\}+[\hat{T}]\{e\}\right)+\{\hat{S}\}+\{f\}=0
$$

where $\epsilon_{L}$ is the linear part of (2), and the elemental matrices and vectors are given by:

$$
\begin{aligned}
& {[\tilde{T}]_{i j}=\int_{\Omega} \vec{W}_{i}^{(1)} \cdot \vec{W}_{j}^{(1)} d \Omega} \\
& {[\hat{T}]_{i j}=\int_{\Omega} \epsilon_{0} \chi^{(3)}\left(\alpha E^{2}+(1-\alpha) g(t) * E^{2}\right) \vec{W}_{i}^{(1)} \cdot \vec{W}_{j}^{(1)} d \Omega}
\end{aligned}
$$

$$
\begin{aligned}
\{\tilde{S}\}_{i} & =\sum_{j} \int_{\Omega} \frac{1}{\mu}\left(\nabla_{D} \times \vec{W}_{i}^{(1)}\right) \cdot\left(\nabla_{B} \times \vec{W}_{j}^{(1)}\right) *\{e\}_{j} d \Omega \\
\{f\}_{i} & =\int_{\Omega} \frac{\partial \vec{J}}{\partial t} \cdot \vec{W}_{i}^{(1)} d \Omega
\end{aligned}
$$

In order to further simplify the $\{\tilde{S}\}$ term it is assumed that the PML conductivity $\sigma$ in (6) is constant within each element. Furthermore, given specific implementation details, additional simplifications are possible by explicitly computing the basis function curls. For example, in the results to follow, linear Whitney 1-forms (edge elements) were used in two dimensions, for which the $\{\tilde{S}\}$ term simplifies considerably:

$$
\{\tilde{S}\}_{i}=\sum_{j} p(t) *\left(\frac{l_{i} l_{j}}{4 A}\right)\{e\}_{j} \triangleq p(t) *[S]\{e\}
$$

in which $l_{i}$ is the edge length associated with $\vec{W}_{i}^{(1)}, A$ is the element area, and $p(t)$ is defined as:

$$
p(t)=\mathcal{F}^{-1}\left\{\left(\frac{1}{s_{y}^{D}}+\frac{1}{s_{x}^{D}}\right)\left(\frac{1}{s_{y}^{B}}+\frac{1}{s_{x}^{B}}\right)\right\} .
$$

where $\mathcal{F}^{-1}(\cdot)$ denotes the inverse Fourier transform. Note that in a non-PML region, $\sigma=0$, and equations (13) and (14) reduce to the usual linear matrix-vector product.

While higher order or 3D basis functions will have more complex expressions, it is in general possible to express them as terms like (14) convolved with a matrix-vector product. Combining equations (13) and (8), the main advantage of the coordinate stretching formulation now becomes clear. Since the PML has only affected the spatial derivatives (corresponding to the $[S]$ matrix), the temporal term which contains all of the material complexity is untouched. As a result, the PML can easily be incorporated into any existing method for complex media, without altering the core structure or functionality. This is in contrast to the traditional uniaxial approach, in which a time-dependent tensor is introduced within the temporal derivative [2]. The result is thus minimal overhead as compared to the non-PML algorithm.

At this stage, a temporal discretization must be applied to (8) in order to proceed. Here, the Newmark- $\beta$ scheme is adopted due to its linear unconditional stability. However, equations (8), (10), and (13) contain three different convolutions, for which update equations must be derived. As mentioned earlier, here the z-transform technique [10]-[12] is adopted for its scalability and simplicity.

Given a convolution, such as that in equation (13):

$$
\{\mathcal{L}\}(t) \triangleq p(t) *[S]\{e\}
$$

one can take the Laplace transform, which has two key advantages. The first is that the convolution is converted to a multiplication, and the second is that $\epsilon_{L}$ and $p(t)$ are generally expressible as the quotient of two polynomials in the frequency-domain. The key step is to then apply a bilinear transform, mapping from the $s$-domain to the discrete $z$ domain:

$$
s \mapsto \frac{2}{\Delta t} \frac{1-z^{-1}}{1+z^{-1}} .
$$


By isolating the $\{\mathcal{L}\}$ term, and leveraging the time shifting property of the z-transform, the expression can be transformed back to the time-domain, yielding an update equation for the convolution:

$$
\begin{aligned}
\{\mathcal{L}\}^{n}=h_{0}[S]\{e\}^{n}+ & \cdots+h_{p}[S]\{e\}^{n-p}- \\
& q_{1}\{\mathcal{L}\}^{n-1}-\cdots-q_{p}\{\mathcal{L}\}^{n-p} .
\end{aligned}
$$

Rather than explicitly storing past field and convolution values, a more efficient approach can be adopted in which quantities are instead accumulated into a set of auxiliary variables $\left\{\mathcal{G}_{\alpha}\right\}^{n}$ as the computation progresses:

$$
\begin{array}{rlrl}
\left\{\mathcal{G}_{\alpha}\right\}^{n} & =h_{\alpha}[S]\{e\}^{n}-q_{\alpha}\{\mathcal{L}\}^{n}+\left\{\mathcal{G}_{\alpha+1}\right\}^{n-1} & & \alpha<p \\
\left\{\mathcal{G}_{\alpha}\right\}^{n} & =h_{\alpha}[S]\{e\}^{n}-q_{\alpha}\{\mathcal{L}\}^{n} & & \alpha=p \\
\{\mathcal{L}\}^{n} & =h_{0}[S]\{e\}^{n}+\left\{\mathcal{G}_{1}\right\}^{n-1} &
\end{array}
$$

Hence, any convolution present in (8) can be replaced with an expression of the form 20,

Combining this procedure with the aforementioned Newmark- $\beta$ method yields the following update equation for the electric field:

$$
\begin{gathered}
\left([K]^{n+1}+\frac{\Delta t^{2}}{4} h_{0}[S]\right)\{e\}^{n+1}= \\
2\left([K]^{n}-\frac{\Delta t^{2}}{4} h_{0}[S]\right)\{e\}^{n}-\left([K]^{n-1}+\frac{\Delta t^{2}}{4} h_{0}[S]\right)\{e\}^{n-1} \\
-\left(\left\{\mathcal{W}_{1}\right\}^{n}+2\left\{\mathcal{W}_{1}\right\}^{n-1}+\left\{\mathcal{W}_{1}\right\}^{n-2}\right) \\
-\frac{\Delta t^{2}}{4}\left(\left\{\mathcal{G}_{1}\right\}^{n}+2\left\{\mathcal{G}_{1}\right\}^{n-1}+\left\{\mathcal{G}_{1}\right\}^{n-2}\right) \\
-\frac{\Delta t^{2}}{4}\left(\{f\}^{n+1}+2\{f\}^{n}+\{f\}^{n-1}\right)
\end{gathered}
$$

where the $\{\mathcal{W}\}$ auxiliary variables are associated with the linear dispersion, $\{\mathcal{G}\}$ with the PML convolution, and $[K]=$ $a_{0}[\tilde{T}]+[\hat{T}]$. Note that the convolution associated with the dispersive Raman nonlinearity occurs within the matrix $[K]$, and that $a_{0}$ is associated with the linear dispersion convolution.

The update equation in 21 is nonlinear due to the implicit dependence of the $[K]$ matrix on $\vec{E}$. This problem can be written of the form $\{F\}(\{x\})=0$, and thus can make use of a nonlinear solver such as Newton-Raphson:

$$
\{x\}_{(k+1)}=\{x\}_{(k)}-[J]^{-1}\{F\}_{(k)}
$$

where $k$ is the iteration number and $[J]$ is the Jacobian matrix, defined as:

$$
[J]_{i j}=\frac{\partial\{F\}_{i}}{\partial\{x\}_{j}} .
$$

The Jacobian for the update equation in 21) was previously derived for nonlinear dispersive problems in $[8]$ and [9]. Luckily, thanks to the use of coordinate stretching, the introduction of a PML has not altered the update equation significantly, and so $[J]$ remains largely unchanged:

$$
\begin{aligned}
& {[J]_{i j}=[K]_{i j}+\frac{\Delta t^{2}}{4} h_{0}[S]_{i j}+} \\
& \int_{\Omega} \frac{1}{E^{n+1}} \frac{\partial \epsilon^{n+1}}{\partial E^{n+1}}\left(\vec{W}_{i}^{(1)} \cdot \vec{E}^{n+1}\right)\left(\vec{W}_{j}^{(1)} \cdot \vec{E}^{n+1}\right) d \Omega
\end{aligned}
$$

where in the present case:

$$
\frac{\partial \epsilon^{n+1}}{\partial E^{n+1}}=2 \epsilon_{0} \chi^{(3)} E^{n+1}\left(\alpha+(1-\alpha) p_{0}\right) .
$$

where the $p_{0}$ term is that associated with the auxiliary variables arising from the nonlinear Raman convolution within the integral in 10 .

\section{RESULTS}

In order to test the FETD MIPML implementation described above, a test problem was devised in which a temporal soliton propagates within a $2 \mathrm{D}$ dielectric slab waveguide. Here, the presence of nonlinearity counteracts anomalous linear dispersion, yielding a pulse which propagates without changing shape or being significantly distorted. Given the presence of linear dispersion, instantaneous nonlinearity, and dispersive nonlinearity, this represents a very general test case.

The waveguide's rectangular domain measured $10 \mu \mathrm{m}$ wide and was composed of three dielectric layers. The center dielectric measured $2 \mu \mathrm{m}$ wide, with the remaining areas being free space. The pulse was excited on the leftmost boundary in the fundamental TM mode, with a modulated hyperbolic secant envelope in time. The pulse envelope had a Full Width at Half Maximum (FWHM) of approximately 52.7 fs and a fundamental frequency of $50 \mathrm{THz}$. Approximately 6 periods of the carrier wave were contained within the pulse.

As in [8], the linear dispersion was modeled as being second-order Lorentz, with a resonant frequency of $30 \mathrm{THz}$, damping factor of $2 \times 10^{11}$, and static/infinite susceptibilities of $6.1 \& 4.7$, respectively. The nonlinear parameters were also modeled, in part, by a Lorentz-type dispersion, with an optical phonon period of $3.36 \times 10^{-14} \mathrm{~s}$, phonon lifetime of $1 \times 10^{-13}$ s, nonlinear susceptibility of $\chi^{(3)}=1.1 \times 10^{-18}$, and $\alpha=0.7$.

Within the PML region, the conductivity, or stretching, is gradually turned on with the following profile, to help minimize numerical reflections resulting from the discretization:

$$
\sigma_{D}=\sigma_{B}=\sigma_{\max }\left(\frac{d}{L}\right)^{4}
$$

where $d$ is the distance into the PML region, $L$ is the length of the PML region, and $\sigma_{\max }$ was set to $3 \times 10^{14}$. However, since the conductivity is assumed constant within each element, the PML region was divided into 20 rectangular sub-regions, yielding a piecewise-constant approximation to the profile in 26. The PML solution was compared to a reference solution obtained from an extended domain with PEC boundaries.

Fig. 1] shows a comparison of the y-component of both signals as measured 10 grid points from the PML interface, in the middle of the guide, with an average element side length of $\approx 0.1 \mu \mathrm{m}$. The two signals are visually indistinguishable, with very little variation or reflection demonstrated by the PML.

To better quantify the reflection error, the reflection coefficient was also computed as follows:

$$
\Gamma(\omega)=20 \log _{10}\left|\frac{\mathcal{F}\left(E_{y}^{e x}\right)-\mathcal{F}\left(E_{y}^{p m l}\right)}{\mathcal{F}\left(E_{y}^{e x}\right)}\right|
$$

in which $E_{y}^{e x}$ is the extended solution, $E_{y}^{p m l}$ is the PML solution, and $\mathcal{F}(\cdot)$ denotes the Fourier transform. 


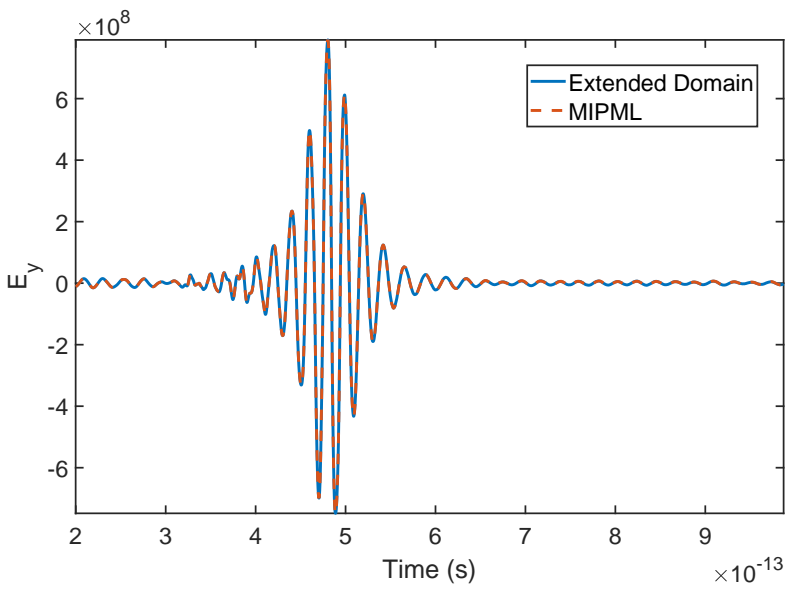

Fig. 1. Comparison of extended domain and MIPML electric fields as a function of time, for a $20 \mu m$ thick PML.

The reflection coefficient so defined is plotted in Fig. 2 as a function of frequency and PML length. Moreover, the normalized frequency spectrum of the incident pulse is also plotted for comparison. For a $20 \mu m$ thick PML, reflection is only $-60 \mathrm{~dB}$ at the pulse's fundamental frequency, representing a reflection of only $0.1 \%$ of the incident wave, or $0.0001 \%$ of the incident power. Over the remaining bandwidth of the signal, the PML also performs well, with an average reflection of $-58.5 \mathrm{~dB}$, and a peak reflection of $-43.1 \mathrm{~dB}$. While very little spectral energy of the pulse is contained in the $30-35 \mathrm{THz}$ region $(\approx 0.5 \%)$, it is worth noting that reflections in this area increase substantially at all thicknesses due to the proximity of the resonance frequency of the linear dispersive medium. This is consistent with prior findings in FDTD implementations [6].

Lastly, it was found that using fewer piecewise-constant slices to approximate (26) yielded higher reflections. For better performance, the number of layers can be further increased to yield a better adiabatic approximation [13].

\section{CONCLUSION}

In conclusion, an FETD implementation of a MIPML has been presented for the truncation of numerical domains containing electrically complex materials, including general combinations of linear dispersion, instantaneous nonlinearity, and dispersive nonlinearity.

By making use of the Newmark- $\beta$ method, as well as the $\mathrm{z}$-transform, and a full nonlinear solver, the resulting scheme is stable, scalable, accurate, and is free from recursiveconvolutions, auxiliary differential equations, and linearizations. Moreover, by leveraging the coordinate-stretching formulation of the PML, the resulting algorithm requires minimal overhead or modification to the underlying method.

The performance of the MIPML was demonstrated by truncating a dielectric slab waveguide in two dimensions, in which a temporal soliton was created. Good absorption was found over the bandwidth of the incident pulse.

Given the large computational burden posed by nonlinear FETD simulations, this represents an important tool not only for emulating infinite domains, but also for reducing computation size, resources, and time.

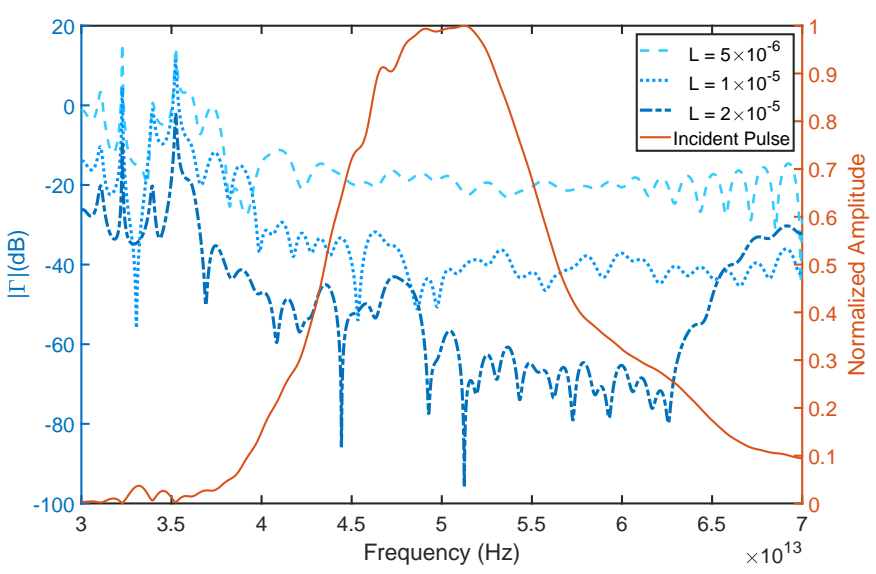

Fig. 2. Reflection coefficient vs frequency for the MIPML.

\section{ACKNOWLEDGMENT}

The authors would like to thank the Natural Sciences and Engineering Research Council of Canada (NSERC) for their support.

\section{REFERENCES}

[1] J.-P. Berenger, "A perfectly matched layer for the absorption of electromagnetic waves," Journal of Computational Physics, vol. 114, no. 2, 1994.

[2] S. D. Gedney, "An anisotropic pml absorbing media for the fdtd simulation of fields in lossy and dispersive media," Electromagnetics, vol. 16, no. 4, 1996.

[3] C. M. Rappaport, "Perfectly matched absorbing boundary conditions based on anisotropic lossy mapping of space," IEEE Microwave and Guided Wave Letters, vol. 5, no. 3, 1995.

[4] A. Giannopoulos, "Unsplit implementation of higher order pmls," IEEE Transactions on Antennas and Propagation, vol. 60, no. 3, Dec 2011.

[5] A. P. Zhao, J. Juntunen, and A. V. Raisanen, "Generalized materialindependent pml absorbers for the fdtd simulation of electromagnetic waves in arbitrary anisotropic dielectric and magnetic media," IEEE Microwave and Guided Wave Letters, vol. 8, no. 2, 1998.

[6] J. Li, L. Mao, J. Miao, C. Guo, and X. Wang, "Efficient pml implementation for truncating nonlinear fdtd lattices based on the z-transform method," in 2009 International Conference on Microwave Technology and Computational Electromagnetics (ICMTCE 2009), Nov 2009, pp. 375-378.

[7] K. J. Blow and D. Wood, "Theoretical description of transient stimulated raman scattering in optical fibers," IEEE Journal of Quantum Electronics, vol. 25, no. 12, pp. 2665 - 2673, Dec. 1989.

[8] D. S. Abraham and D. D. Giannacopoulos, "A convolution-free mixed finite-element time-domain method for general nonlinear dispersive media," IEEE Transactions on Antennas and Propagation, 2018, early Access.

[9] — "A convolution-free finite-element time-domain method for the nonlinear dispersive vector wave equation," IEEE Transactions on Magnetics, 2018, submitted for review.

[10] A. Akbarzadeh-Sharbaf and D. D. Giannacopoulos, "A stable and efficient direct time integration of the vector wave equation in the finiteelement time-domain method for dispersive media," IEEE Transactions on Antennas and Propagation, vol. 63, no. 1, pp. 314-321, Jan. 2015.

[11] N.-X. Feng, J.-X. Li, and X.-M. Zhao, "Efficient fdtd implementations of the higher-order pml using dsp techniques for arbitrary media," IEEE Transactions on Antennas and Propagation, vol. 61, no. 5, Jan 2013.

[12] N. Feng, Y. Yue, and Q. H. Liu, "Direct z-transform implementation of the cfs-pml based on memory-minimized method," IEEE Transactions on Microwave Theory and Techniques, vol. 63, no. 3, Jan 2015.

[13] A. F. Oskooi, L. Zhang, Y. Avniel, and S. G. Johnson, "The failure of perfectly matched layers, and towards their redemption by adiabatic absorbers," Optics Express, vol. 16, no. 15, pp. 11 376-11 392, Jul 2008. 